\title{
Electrical modulation of emissivity
}

\author{
S. Vassant, ${ }^{1}$ I. Moldovan Doyen, ${ }^{1}$ F. Marquier, ${ }^{1}$ F. Pardo, ${ }^{2}$ U. Gennser, ${ }^{2}$ A. Cavanna, ${ }^{2}$ \\ J. L. Pelouard, ${ }^{2}$ and J. J. Greffet ${ }^{1, a)}$ \\ ${ }^{1}$ Laboratoire Charles Fabry, Institut d'Optique, CNRS-UMR8501, Université Paris-Sud, Campus \\ Polytechnique, RD 128, 91127 Palaiseau cedex, France \\ ${ }^{2}$ Laboratoire de Photonique et Nanostructures, CNRS-UPR20, Route de Nozay, 91460 Marcoussis, France
}

(Received 15 October 2012; accepted 13 February 2013; published online 1 March 2013)

\begin{abstract}
We demonstrate that it is possible to modulate the thermal emission through an electrical modulation of the emissivity. The basic idea is to design a device where absorption is due to a resonant phenomenon. If the resonance can be electrically controlled, then absorption and, therefore, thermal emission can be controlled. We demonstrate this general concept using $\mathrm{THz}$ resonant absorption by surface phonon polaritons coupled through a gold grating. In our device, absorption is mostly due to a surface phonon mode confined in a single quantum well (QW). The $\mathrm{THz}$ emissivity is electrically controlled by controlling the quantum well refractive index using a gate voltage to modulate the electron density. This paves the way to electrically modulated incandescent sources. (C) 2013 American Institute of Physics. [http://dx.doi.org/10.1063/1.4793650]
\end{abstract}

Incandescent sources such as light bulbs have been widely used for lighting applications over a very wide range of frequencies from UV to THz. However, such sources suffer from a number of limitations, such as the non-directional emission over a broad spectrum, a low energy conversion efficiency, and a modulation rate restricted to a few tens of $\mathrm{Hz}$. Hence, for many applications, incandescent light sources cannot compete with light emitting diodes. Yet, incandescent sources are often the only option in the infrared (IR). Indeed, it is difficult to develop an efficient light emitting diode because the spontaneous emission rate is proportional to $\nu^{3}$ where $\nu$ is the emitted frequency. Improving the performances of spontaneous emission by incandescent sources in the IR is thus required. The broad emission spectrum and the lack of directivity are a direct consequence of the lack of coherence of the source. While it has been assumed for a long time that an incandescent source is intrinsically incoherent, many works ${ }^{1-6}$ have now reported the possibility of designing spatially coherent sources that produce collimated beams. There has also been an important development of systems, ${ }^{7-16}$ demonstrating temporally coherent thermal sources producing narrow band emission. In this paper, we address the issue of electrical modulation of the emitted intensity. Presently, incandescent sources can be modulated by modulating the temperature. While heating a filament can be fast, the cooling time is limited by heat transport. In practice, it is hardly possible to modulate faster than $100 \mathrm{~Hz}$. In this paper, we demonstrate an alternative concept of electrical control of emitted flux, which could lead to fast modulation of the thermal emission. It is wellknown that the emitted radiation is characterized by a specific intensity $I_{\nu}^{(e)}(T, \theta)$ given by

$$
I_{\nu}^{(e)}(T, \theta)=\epsilon(\nu, \theta) I_{\nu}^{(B B)}(T),
$$

where $\theta$ is the direction of emission and $T$ the temperature of the source. $\epsilon(\nu, \theta)$ is the emissivity, which is equal to the

\footnotetext{
${ }^{\text {a) }}$ Author to whom correspondence should be addressed. Electronic address: jean-jacques.greffet@institutoptique.fr.
}

absorptivity according to Kirchhoff's law. ${ }^{17}$ Hence, it is seen that the emitted flux can be modulated by modulating the emissivity $\epsilon(\nu, \theta)$ instead of modulating the temperature. ${ }^{18}$ The basic idea to design such a system is to start from a nonabsorbing system, so that the emissivity is low or zero. By introducing a resonant absorbing structure, it is possible to choose the absorption frequency. In order to achieve an active system, the resonance has to be controlled externally. If the resonance can be turned off electrically, then it is possible to control the absorptivity and, therefore, the emissivity of the source.

In order to demonstrate this general idea, we use a resonant absorption due to the excitation of surface phonon polaritons coupled through a grating. The system is depicted in Fig. 1. It consists in a multilayer composed of an n-doped GaAs substrate (d), a spacer (c), a $22 \mathrm{~nm}$-thick GaAs quantum well (QW) (b) embedded in $\mathrm{Al}_{0.33} \mathrm{Ga}_{0.67} \mathrm{As}$ barriers, and finally a gold grating (a). Further details on the device dimensions are indicated in Fig. 1. Electrons in the QW are supplied by a Si delta-doping of $9.85 \times 10^{11} \mathrm{~cm}^{2}$ situated at $40 \mathrm{~nm}$ from the QW.

Reference 19 has shown that this device presents a resonant absorption at a particular wavelength close to $34.3 \mu \mathrm{m}$ and at ambient temperature. The absorption mechanism is the following. First, it has to be noticed that an electromagnetic mode exists at this wavelength in the QW film. This mode consists in a strong coupling between surface phonon polaritons, propagating along the GaAs/AlGaAs interfaces with a large wavevector whose typical value is $1 \mu \mathrm{m}^{-1}$. Its frequency is close to the GaAs longitudinal-phonon frequency, so that the dielectric constant is close to zero. Due to the continuity of $\epsilon E_{z}$ at the interfaces GaAs/AlGaAs, the z-component of the electric field $E_{z}$ is orders of magnitude larger in the QW than in the barriers. It follows that the mode is highly confined in the QW. It has been called epsilon near zero (ENZ) mode in Refs. 19 and 20.

The grating parameters have been optimized to couple incident propagating waves to this ENZ mode. Its resonant excitation leads to an absorption peak QW at $34.3 \mu \mathrm{m}$. Note 


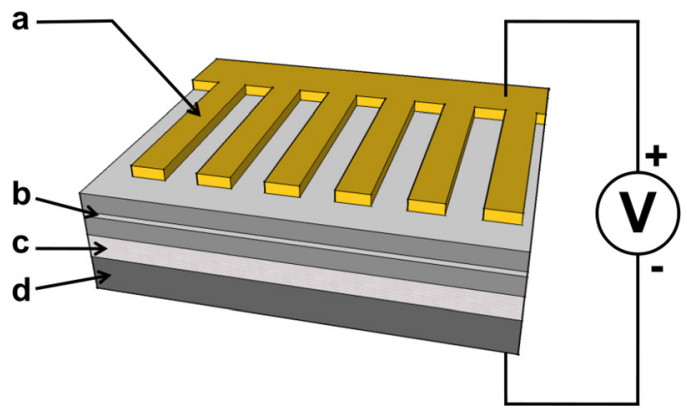

FIG. 1. Sketch of the emitting system. A gold grating (a) is deposited on a $\mathrm{AlGaAs} / \mathrm{GaAs}$ heterostructure consisting in a single $22 \mathrm{~nm}$-thick GaAs quantum well (b) embedded in $\mathrm{Al}_{0.33} \mathrm{Ga}_{0.67} \mathrm{As}$ barriers $(100 \mathrm{~nm}$ and $90 \mathrm{~nm}$ in thickness). The grating is characterized by a $4 \mu \mathrm{m}$ period, $66 \%$ filling factor, and $200 \mathrm{~nm}$ thickness. A $1.3 \mu$ m-thick $\mathrm{Al}_{0.5} \mathrm{Ga}_{0.5} \mathrm{As}$ spacer (c) separating the barriers and an n-doped GaAs substrate (d) allows optimizing the absorption in the single quantum well. A gate voltage $\mathrm{V}$ can be applied between the grating and the substrate leading to a change in the emissivity of the structure. The surface of the obtained device is about $5 \times 5 \mathrm{~mm}^{2}$.

that the absorption is due to the excitation of surface phonon polaritons that further decay into heat in the single quantum well so that the losses are highly localized. This process is analogous to absorption of light by a metallic grating due to resonant excitation of surface plasmons. It is very different from absorption due to intersub-band transitions (ISBTs) of electrons in a QW.

Reflectivity (R) measurements demonstrating this effect are reported in Ref. 19. We now discuss how the absorption can be modulated electrically. We designed the thickness of the QW in order to generate an ISBT in resonance with the surface phonon polaritons at $34.3 \mu \mathrm{m}$. When electrons are injected in the QW, they produce a resonant contribution to the dielectric constant, which then deviates from zero. As a result, the ENZ enhancement of the electric field can be suppressed because the dielectric constant is no longer close to zero. To change the number of electrons in the QW, we apply a voltage between the metallic grating and the doped substrate. It follows that the resonant absorption can be turned off when increasing the number of electrons in the QW. In summary, the resonant absorption effect can be switched off and on using an external electrical command.

According to Kirchhoff's law, the emissivity is simply given by $1-\mathrm{R}$ in the case of an opaque body. As a result, the emissivity of such a structure is expected to be modulated using an external voltage, yielding a modulated thermal emission. However, for emission applications, one needs to increase significantly the temperature so that the optical properties change. As the system is extremely sensitive on the value of the dielectric constant because of the resonant character of the absorption, it is not obvious that the electrical control of the emissivity is still a valid concept at high temperature. Here, we report an experimental demonstration of the electrical control of the thermal-emission. In this paper, the same sample as in Ref. 19 is used as a thermal emitter. A sketch of the experimental setup is seen in Fig. 2. It consists mainly in a Fourier transform spectrometer (BRUKER, Vertex 70) in which both a Si beamsplitter and a pyroelectric (DTGS) detector are chosen according to their sensitivity in the terahertz range. Thermal emission due to the excitation of surface phonon polariton in the QW is

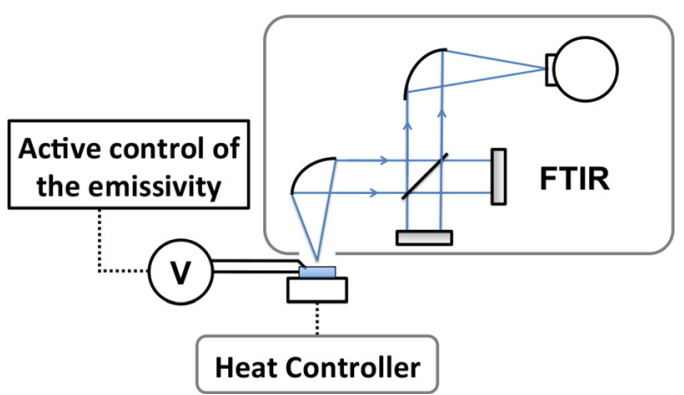

FIG. 2. Experimental setup used to characterize the modulated thermal emission of the structure. The temperature of the sample is set to $70^{\circ} \mathrm{C}$. A DC gate voltage ranging from $-400 \mathrm{mV}$ to $400 \mathrm{mV}$ is applied and allows an active control of the emissivity. The radiation is spectrally analyzed using an infrared Fourier transform spectrometer (FTIR) with a mylar beamsplitter and a DTGS detector.

expected to be isotropic. The sample is thus optically conjugated to the detector without any spatial filtering, so that the whole thermal emission in the numerical aperture (roughly a $10^{\circ}$-wide cone) of the spectrometer is detected, allowing the best signal level from the source. A slight overpressure of dry air in the spectrometer is used to avoid water absorption peaks in the signal. The temperature of the sample holder is set to $70^{\circ} \mathrm{C} \pm 1^{\circ} \mathrm{C}$ with a heat controller. The gate voltage is provided by a stabilized source (AGILENT B2902A). To enhance the low signal to noise ratio, each spectrum is taken after averaging 2000 interferograms. A $100^{\circ} \mathrm{C}$ blackbody (CI SYSTEMS) is used as a reference to obtain emissivity spectra following the procedure described in Ref. 2 .

Emission spectra are plotted in Fig. 3 for three different gate voltages. It is seen that the thermal emission around $34.5 \mu \mathrm{m}$ is changed when applying a voltage. A negative voltage leads to a higher absorption/emissivity. We obtain the maximum emissivity for $-400 \mathrm{mV}$ and the minimum emissivity for $+400 \mathrm{mV}$. In the latter case, the electron density in the GaAs film is limited by the onset of a leakage current and reaches thus a maximum, whereas in the first case, the QW is fully depleted. In both cases, increasing more the absolute value of the voltage does not change the GaAs dielectric constant and thus the emissivity. As expected, the emissivity at $70^{\circ} \mathrm{C}$ is not equal to $1-R$ at ambient temperature reported in Ref. 19: varying the voltage, the emissivity relative variations are about $9 \%$, when the reflectivity relative variations are

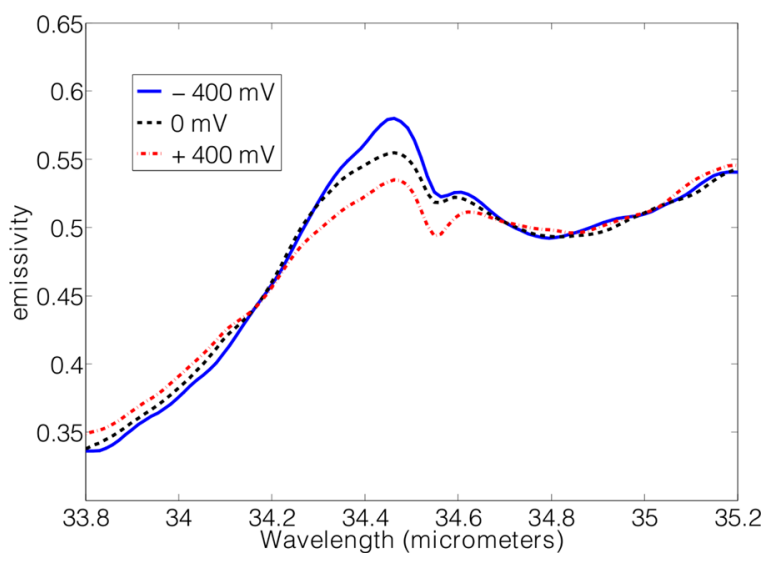

FIG. 3. Emissivity spectrum of the sample at 3 different gate voltages. The emissivity is varying with the gate voltage at the resonant surface phonon polariton wavelength in the quantum well. 
about $50 \%$. Increasing the device's temperature, the emissivity peak becomes smaller and wider (not shown here). Hence the temperature-related variations of the semiconductors dielectric constants lead to a significant reduction of the absorption/emissivity resonance. However, electrical control of the thermal emission without varying the sample temperature has been demonstrated. The modulation bandwidth cannot be explored with the currently available DTGS detector and is left for future work using THz superconducting detectors.

In summary, we have reported thermal-emission measurements showing a device where the emissivity can be electrically controlled. This can be used to actively modulate thermal emission. Since the modulation speed is driven by the dynamics of the resonant system and not by the temperature dynamics, this work paves the way to fast thermal modulation of incandescent sources.

This research described here has been supported by RTRA through the project PAO 2008-057T, ANR Contract LAPSUS ANR-07-NANO-043, and the region Ile de France through a C'nano IdF project. We thank N. Bardou, C. Dupuis, L. Ferlazzo, and E. Cambril for their help in the clean room. We thank R. Haidar and J. Jaeck from ONERA for lending instruments (Blackbody and Si beamsplitter).

${ }^{1}$ J. J. Greffet, R. Carminati, K. Joulain, J. P. Mulet, S. P. Mainguy, and Y. Chen, Nature (London) 416, 61 (2002).
${ }^{2}$ F. Marquier, K. Joulain, J. P. Mulet, R. Carminati, J. J. Greffet, and Y. Chen, Phys. Rev. B 69, 155412 (2004).

${ }^{3}$ M. Laroche, C. Arnold, F. Marquier, R. Carminati, J. J. Greffet, S. Collin, N. Bardou, and J. L. Pelouard, Opt. Lett. 30, 2623 (2005).

${ }^{4}$ N. Dahan, A. Niv, G. Biener, Y. Gorodetski, V. Kleiner, and E. Hasman, Phys. Rev. B 76, 045427 (2007).

${ }^{5}$ C. Fu and Z. M. Zhang, Front. Energy Power Eng. China 3, 11 (2009).

${ }^{6}$ C. Arnold, F. Marquier, M. Garin, F. Pardo, S. Collin, N. Bardou, J. L. Pelouard, and J. J. Greffet, Phys. Rev. B 86, 035316 (2012).

${ }^{7}$ J. Drevillon and P. Ben-Abdallah, J. Appl. Phys. 102, 114305 (2007).

${ }^{8}$ B. J. Lee, L. P. Wang, and Z. M. Zhang, Opt. Express 16, 11328 (2008).

${ }^{9}$ K. Ikeda, H. T. Miyazaki, T. Kasaya, K. Yamamoto, Y. Inoue, K. Fujimura, T. Kanakugi, M. Okada, K. Hatade, and S. Kitagawa, Appl. Phys. Lett. 92, 021117 (2008).

${ }^{10}$ I. Puscasu and W. L. Schaich, Appl. Phys. Lett. 92, 233102 (2008).

${ }^{11}$ E. Rephaeli and S. Fan, Opt. Express 17, 15145 (2009).

${ }^{12}$ N. Liu, M. Mesch, T. Weiss, M. Hentschel, and H. Giessen, Nano Lett. 10, 2342 (2010)

${ }^{13}$ X. Liu, T. Tyler, T. Starr, A. F. Starr, N. M. Jokerst, and W. J. Padilla, Phys. Rev. Lett. 107, 045901 (2011).

${ }^{14}$ P. Bouchon, C. Koechlin, F. Pardo, R. Haidar, and J. L. Pelouard, Opt. Lett. 37, 1038 (2012).

${ }^{15}$ M. De Zoysa, T. Asano, K. Mochikuzi, A. Oskooi, T. Inoue, and S. Noda, Nat. Photonics 6, 535-539 (2012).

${ }^{16}$ S. Thongrattanasiri, F. H. L. Koppens, and F. J. Garcia de Abajo, Phys. Rev. Lett. 108, 047401 (2012).

${ }^{17}$ J. J. Greffet and M. Nieto-Vesperinas, J. Opt. Soc. Am. A 15, 2735 (1998).

${ }^{18}$ J. J. Greffet, Nature (London) 478, 191 (2011).

${ }^{19}$ S. Vassant, A. Archambault, F. Marquier, F. Pardo, U. Gennser, A. Cavanna, J. L. Pelouard, and J. J. Greffet, Phys. Rev. Lett. 109, 237401 (2012).

${ }^{20}$ S. Vassant, J. P. Hugonin, F. Marquier, and J. J. Greffet, Opt. Express 20, 23971 (2012). 